Check for updates

New York

Cite this as: $B M J 2022 ; 376: 0524$ http://dx.doi.org/10.1136/bmj.0524 Published: 28 February 2022

\title{
Covid-19: CDC relaxes rules on mask wearing
}

\section{Janice Hopkins Tanne}

The US Centers for Disease Control and Prevention (CDC) has relaxed its rules on mask wearing, with around $70 \%$ of Americans no longer required to wear a face covering indoors.

The new rules show the CDC's focus is on minimising serious disease, lessening the strain on hospitals, and protecting the most vulnerable.

"The overall risk of severe disease is now generally lower," CDC director Rochelle Walensky told a press briefing on 25 February. The CDC continued to advise people to be vaccinated and boosted and said they could continue to wear masks if they chose.

The change in guidelines comes as many communities have ended mask mandates and many people have stopped wearing masks. The new guidelines mean that students need to wear masks only in high risk areas. Vaccines are approved for children aged 5 and older.

Covid-19 cases in the US have been decreasing. Over the past two weeks, cases have dropped by $63 \%$, hospital admissions by $44 \%$, and deaths by $23 \%$, according to the New York Times coronavirus tracker. ${ }^{1}$ But only about $65 \%$ of Americans are fully vaccinated.

Walensky said, "We need to give people a break from things like mask wearing when our levels are low." But, she said, the CDC would turn to stronger guidelines "should things get worse in the future."

The CDC said its decision was based on local conditions in each of the nation's 3141 counties. It published a colour coded map for guidance, which codes counties as red (high levels of covid-19), yellow (medium), or green (low). ${ }^{2}$ About $62 \%$ of US counties, in which about $72 \%$ of Americans live, are now considered low or medium risk areas. ${ }^{3}$ Previously, the $\mathrm{CDC}$ recommended that people wear masks in areas of medium and high transmission, about 95\% of all counties. Under the new rules, Boston, Chicago, New York, and Washington, DC, are now low risk areas, Los Angeles is a medium risk area, and Miami and Dallas are high risk areas.

The CDC based its judgment on the number of cases of covid-19 that require hospital admission and the percentage of hospital beds occupied by patients with covid-19 in each county. The colour ratings may change over time as the pandemic changes locally.

People in green or yellow areas no longer need to wear masks indoors, including students in schools. People at higher risk-for example those who are immunocompromised-were told to consult their healthcare providers for advice on mask wearing.

Masks are still required on public transport and at railway and bus stations, airports, and on aeroplanes.

Walensky said that the nation was "now in a stronger place" and the risk of severe disease was now generally lower. She said the US had more tools to protect against covid-19, including vaccinations, boosters, broader access to testing, high quality masks, new treatments, and improved ventilation.

Some 400 public health researchers and practitioners posted an open letter saying it was too soon to lift mask mandates and asked officials to re-evaluate indoor masking in schools. They pointed out that more than 12 million children had been infected with covid-19, some two million in January 2022 alone, and more than 900 children have died from covid-19. Infected children can bring the virus home to their parents and to vulnerable family members, they said. Indoor mask mandates are effective at reducing transmission. Masking in schools should be continued, they added. 4

1 New York Times coronavirus virus in the US: latest map and case count. February 2022. www.nytimes.com/interactive/2021/us/covid-cases.html.

2 Centers for Disease Control and Prevention. Covid-19 by county. 25 February 2022. www.cdc.gov/coronavirus/2019-ncov/your-health/covidby-county.html.

3 Mandavilli A. New CDC guidelines suggest 70 percent of Americans can stop wearing masks. New York Times. 25 February 2022. www.nytimes.com/2022/02/25/health/cdc-mask-guidance.html.

4 Open Letter: Public health researchers and practitioners urge elected officials to revaluate the end of school mask mandates.https://medium.com/@pubhealthschoolmasks/open-letter-public-health-school-mask-mandatese3a7071f3b26.

This article is made freely available for personal use in accordance with BMJ's website terms and conditions for the duration of the covid-19 pandemic or unti otherwise determined by BMJ. You may download and print the article for any lawful, non-commercial purpose (including text and data mining) provided that all copyright notices and trade marks are retained. 